\title{
Efficacy of Manual Liquid Based Cytology over Conventional Cytology in Oral Squamous Cell Carcinoma
}

\author{
Sudhir Mishra ${ }^{1}$, Jagadish Hosmani ${ }^{1}$, Nandini Manoli ${ }^{2}$, Ramakant Nayak ${ }^{1}$, Manjunath G. V. ${ }^{2}$ \\ ${ }^{1}$ Department of Oral Pathology and Microbiology, Rajiv Gandhi University, Belgaum, karnataka, India \\ ${ }^{2}$ Department of Pathology, JSS University, Mysore, karnataka, India
}

Email address:

mishrasudhir5050@gmail.com (S. Mishra),jhosmani@gmail.com (J. Hosmani), nanda-dr05@yahoo.com (N. Manoli), ramakantnayak30@hotmail.com (R. Nayak),manjugatti@yahoo.com (Manjunath G. V.)

\section{To cite this article:}

Sudhir Mishra, Jagadish Hosmani, Nandini Manoli, Ramakant Nayak, Manjunath G. V.. Efficacy of Manual Liquid Based Cytology over Conventional Cytology in Oral Squamous Cell Carcinoma. Science Journal of Clinical Medicine. Special Issue: Latest Different Concepts of Gynaecology. Vol. 4, No. 4-1, 2015, pp. 11-15. doi: 10.11648/j.sjcm.s.2015040401.13

\begin{abstract}
Context/Background: Oral cytology has come long way from its primitive Papanicolau days. Liquid Based cytology has shown significant advantages over conventional exfoliative cytology. However, LBC requires expensive automated devices and materials which might not be affordable for many cytopathological laboratories in countries with limited resources. Manual liquid Based Cytology (MLBC) is a technique that enables cells to be suspended in a monolayer and thus improves detection of lesions and improvement of adequacy. Aim: To study and evaluate the diagnostic efficiency and reliability of MLBC in comparison with conventional PAP smear (CPS) of oral squamous cell carcinoma. Materials and Methods: Two smear were prepared from 50 patients, clinically diagnosed with Oral Squamous Cell Carcinoma. Each smear was subjected to MLBC and CPS methods. The slides were evaluated by two pathologists for the staining characterstics of nucleus and cytoplasm. The diagnostic efficiency of each smear was evaluated by comparing the cytological diagnosis of each method with histopathological diagnosis. Results: Increased detection rate with MLBC was $29.41 \%$. Identifying cellular atypia by MLBC was more sensitive (44\%) compared to CPS (34\%) with similar specificity (100\%). The percentage agreement by the two methods was $77.28 \%$. Conclusion: MLBC is an easy, cost effective technique comparable to CPS; however, it warrants further study in its potential application in screening of oral precancer and cancer.
\end{abstract}

Keywords: Conventional Pap Smear, Manual Liquid Based Cytology, Oral Cytology, Oral Squamous Cell Carcinoma

\section{Introduction}

At the world level, head and neck cancer is the sixth most common cancer. ${ }^{1}$ Oral squamous cell carcinoma (OSCC) accounts for about $40 \%$ of head and neck and $90-95 \%$ of oral malignancies. ${ }^{2,3}$ Detecting oral malignant and potentially malignant lesions in early stages dramatically affects survival rates. Unfortunately, $50 \%$ of patients have regional or distant metastases at the time of diagnosis, which reflects a significant diagnostic delay. ${ }^{4,5}$ Five-year survival is about $76 \%$ to $80 \%$ if diagnosis is performed in stage 1 and 2 . Late diagnosis in stage 3 and 4 can decrease this value to $41 \%$ and $9 \%$ respectively. ${ }^{2,3,6,7}$ Despite recent advances in treatment modalities, the survival rate of patients with oral cancer has not significantly improved. Therefore, new avenues are being explored in the era of evolving personalized patient management by early detection. Oral cytology has come a long way from its primitive Papanicolaou days. ${ }^{8,9}$ It has made major strides in its eventful development. Conventional Pap Smears (CPS) sensitivity reduces to less than 50\% when there is presence of obscuring blood, inflammation or thick areas of overlapping epithelial cells. ${ }^{10,11}$ These problems with the CPS, gave rise to the advanced technologies, Liquid based cytology. Liquid-based cytology (LBC), since its inception in the 1990s, has shown significant advantages over conventional exfoliative cytology. Although conventional cytology is useful when diagnosing oral precancer \& cancer, LBC gives better results, as it not only enhances both sensitivity and specificity, but also provides material for further investigation (AgNORs, DNA, immunohistochemistry, etc.). ${ }^{8,12}$ In most published series, LBC allows a good inter-observer reproducibility. ${ }^{8}$ However, LBC requires expensive automated devices and materials, which might not be affordable for many cytopathology laboratories in countries with poor resources. ${ }^{8,9}$ 
On the other hand, Manual Liquid Based Cytology (MLBC) introduced by Maskem et al in 2001 is a technique that enables cells to be suspended in a monolayer and thus improves detection of precursor lesions and improvement of specimen adequacy. Many studies have shown that with proper training, MLBC results in a higher diagnostic yield than traditional cervical smears. ${ }^{8,13}$

Therefore the overall aim of our study was to compare efficacy of manual liquid based cytology over conventional cytology in screening of oral squamous cell carcinoma. Specific objectives of the study were: a) Compare the morphological view (nuclear and cellular parameter) in oral squamous cell carcinoma according to CPS and MLBC. b) Compare the validity of two methods in terms of sensitivity.

\section{Materials and Methods}

The study included 50 patients in the age range of 20 to 70 years with clinical diagnosis of oral cancer. A plastic spatula and wooden ice cream stick was used to collect the samples. Spatula was rotated against the lesion. Wooden stick material from one side of the spatula was spread onto a clean glass slide and fixed by bio-spray for conventional method. The material from plastic spatula was dipped into a bottle with fixative prepared in our laboratory. The specimens were subjected to two methods for morphological diagnosis namely Conventional Pap Smear (CPS) and Manual Liquid Based Cytology (MLBC).

\subsection{CPS Method}

This method included the standard procedure of usual staining of the glass slides with the spread smear. Rapid pap method of staining was used.

\subsection{MLBC Method}

We report here an indigenous method which is specific to our laboratory using chemicals available in the laboratory, a simple equipment, fixative and polymer solution prepared by us, thus making it a low cost manual method of oral cancer Pap smear screening. The method was accomplished in the following steps of processing.

The material collected in the liquid fixative (containing sodium chloride, sodium citrate, $10 \%$ formalin and isopropyl alcohol) was further processed after a minimum duration of 24 hours. The procedure involved first the mixing of the sample properly before transferring it to a clean test tube and centrifuging it at 1,000 rounds per minute (rpm) for 5 minutes. The supernatant was then decanted. Two millilitre of polymer solution containing agarose, polyethylene glycol, poly-l-lysine and alcohol was added to the deposit. This was further centrifuged at 2,000 rpm $(600-800 \mathrm{~g})$ for 5-10 minutes The supernatant was discarded. Two millilitre of phosphate buffer solution is added to the deposit. The supernatant was discarded and from the deposit smear was made on a clean glass slide using a Pasteur pipette. The prepared slides were fixed by drying it room temperature for 2-3 hrs. The slides were further fixed by dipping it in $95 \%$ alcohol for 15 minutes and stained with rapid pap stain.

\section{Observation}

The smears were studied by two independent observers and cytological smear are categorized into one of five classes.

Table 1. Cytology Categories into 5 Classes. ${ }^{14}$

\begin{tabular}{ll}
\hline CATERGORIES & RESULTS \\
\hline CLASS I & NORMAL \\
CLASS II & ATYPICAL \\
CLASS III & INDETERMINATE \\
CLASS IV & SUGGESTIVE FOR CANCER \\
CLASS V & POSITIVE OF CANCER \\
\hline
\end{tabular}

\section{Statistical Analysis}

The frequency distribution of leading morphological features was worked out to compare the same according to the two methods under study. Increased detection rate (IDR) was calculated as following, $\mathrm{IDR}=((\mathrm{Pm}-\mathrm{Pc}) / \mathrm{Pc}) * 100$, where, $\mathrm{Pm}$ is the number of positive cases through MLBC and $\mathrm{Pc}$ is the same through CPS.

Subsequently, in order to compare the validity of CPS and MLBC in the diagnosis of oral cancer, sensitivity of the same were estimated considering the histopathological examination (HPE) as the gold standard method.

\section{Results}

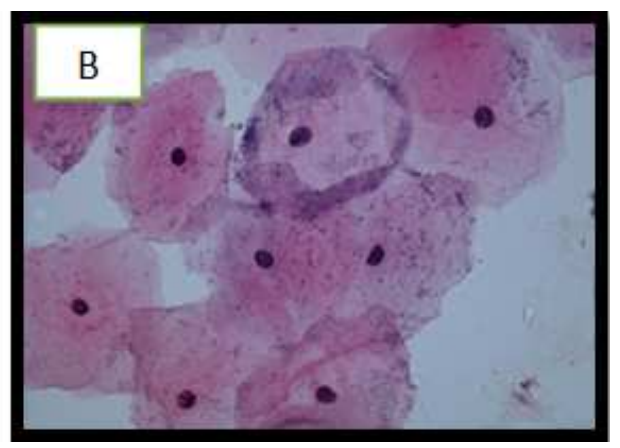

Figure 1. Normal Smear by both CPS and MLBC Methods. A) CPS (40x) B) MLBC (40x). 
Cellularity was adequate in all of the MLBC cases whereas it was unsatisfactory in many CPS cases. The background was observed to be clean in all cases of MLBC which was not the case in majority of CPS. There was artefacts were present in most CPS samples. Architectural and cellular morphologic changes were present in most of CPS samples. Inflammatory infiltrate were prominently present in CPS but decreased in MLBC cases. Nuclear changes were very clear by MLBC, but not so clear by CPS. Diagnostic features of 50 cases according to both CPS and MLBC were divided (table 2).

Table 2. Diagnostic features of 50 cases according to both CPS and MLBC

\begin{tabular}{llll}
\hline SL & \multirow{2}{*}{ CATERGORIES } & CPS & MLBC \\
NO & CLASS I & 32 & 27 \\
\hline 1. & CLASS II & 6 & 6 \\
2. & CLASS III & 8 & 12 \\
3. & CLASS V & 3 & 4 \\
4. & UNSTATISFACTORY & 1 & 1 \\
\hline
\end{tabular}

Validity of the two methods

To compare the validity of the two methods, we estimated sensitivity of the two methods considering HPE as the gold standard. In the diagnosis of atypical cell, MLBC was more sensitive than CPS (44\% vs. $34 \%$ ). (Table 3 )

Table 3. Sensitivity of $M L B C$ and $C P S$.

\begin{tabular}{ll}
\hline CATERGORIES & SENSITIVITY (\%) \\
\hline Conventional pap smear & 34 \\
Manual liquid based cytology & 44 \\
\hline
\end{tabular}

\section{Discussion}

Oral cytology appeared to be a promising diagnostic tool as it was thought to have potential for early detection of malignant lesions. The issue of whether oral cytology could be applicable for mass population screening is somewhat unsettled, although the majority opinion seems to be that it was not practical at that time. ${ }^{15}$ Over a period of time, as the field of oral cytology started to grow, many investigators including Montgomery and von Haam experienced the limitations of oral cytology and therefore felt the need for improvements. The conventional pap smear has been utilized for oral cancer screening for many years. Despite being credited for early detection, conventional papanicolaou smear (CPS) has its limitation. False negatives in CPS may be related to inadequate sampling, inadequate transfer of the sample onto the glass slide or deficiencies in the microscopic assessment of the slide. ${ }^{8,13}$ Liquid based cytology has been developed to address the sampling problems of conventional Pap smear. ${ }^{16}$ This technique commonly used in developed countries, but may not be affordable in the developing countries due to paucity of resources. To overcome these problems, a new slide preparation method namely the Manual Liquid Based Cytology (MLBC) was introduced by (Maksem et al., 2001). ${ }^{8}$ The present work was done to evaluate the Manual liquid based cytology and to compare the sensitivity of the same with conventional Pap smear.

Manual liquid based cytology (MLBC) are cost effective, improves detection of suspicious lesions and specimen adequacy. Although a clinician may have excellent collection and sampling technique, only approximately $20 \%$ of the cells collected are smeared on the glass slide in CPS. ${ }^{16,17,18}$ In our study the MLBC method was found to be superior to the conventional pap smear.

In most published series liquid based cytology was found to be more sensitive than conventional pap smear. ${ }^{8,9,19}$

The present study showed that MLBC is more sensitive than conventional pap smear (44 vs 34) for atypical cell. Our study found increased detection rate with MLBC as compare to CPS.

There were several studies carried out to compare specimen adequacy and diagnostic agreement between liquid - based preparations and conventional smears in oral lesions. Study conducted by Hayama et al. show LBC demonstrated $41 \%$ overall improvement in smear thickness and $66 \%$ in cell distribution, and a reduction in cell overlying and presence of blood than CPS. It showed an overall improvement on sample preservation, specimen adequacy, visualization of cell morphology and reproducibility. ${ }^{9}$ Similar study conducted by Navon et al show LBC has higher sensitivity than conventional cytology ( 95.1 vs 85.7 ) $\%$ and higher specificity (95.9 vs 99.0$) \%$ respectively. ${ }^{20}$

A study by Nandini et al found MLBC was more sensitive in diagnosis LSIL (low grade squamous intraepithelial lesion) and more specific in the diagnosis of inflammation than CPS. ${ }^{8}$ Result shown by Nandini et al. is similar to our study.

Manual method of liquid based cytology which we are following is an inexpensive, cost effective method of LBC which we have adapted and are comparing it with conventional pap smears (CPS) for its adequacy and utility The other advantages of MLBC method is that the residual specimens can be used for ancillary testing like imunocytochemistry by cell block.

In conclusion, the low cost manual liquid based cytology method of oral screening was found to be better than the standard commercial method. It also over comes the limitations of CPS. However, it warrants further study in its potential application in screening of oral cancer.

\section{References}

[1] Fedele, S. 2009. Diagnostic aids in the screening of oral cancer. Head Neck Oncology. 1(5): doi:10.1186/1758-3284-1-5.

[2] Neville, B.W., D.D. Damm, C.M. Allen, and J.E. Bouquot. 1995. Oral \& maxillofacial pathology. Vol. 620: Saunders Philadelphia.

[3] Pektas, Z.Ö., A. Keskin, Ö. Günhan, and Y. Karslioglu. 2006. Evaluation of nuclear morphometry and DNA ploidy status for detection of malignant and premalignant oral lesions: quantitative cytologic assessment and review of methods for cytomorphometric measurements. J Oral Maxillofacsurg64 (4):628-635 
[4] Fedele S. Diagnostic aids in the screening of oral cancer. Head Neck Oncol. 2009;1:5

[5] Acha A, Ruesga MT, Rodríguez MJ, Martínez de Pancorbo MA, Aguirre JM. Applications of the oral scraped (exfoliative) cytology in oral cancer and precancer. Med Oral Patol Oral Cir Bucal.2005;10:95-102.

[6] Mashberg, A., and L.J. Feldman. 1988. Clinical criteria for identifying early oral and oropharyngeal carcinoma: Erythroplasia revisited+. Am J Surg 156 (4):273-275.

[7] Maraki, D., J. Becker, and A. Boecking. 2004. Cytologic and DNA cytometric very early diagnosis of oral cancer. J Oral Pathol Med 33 (7):398-404.

[8] Nandini NM, Nandish SM, Pallavi P, Akshatha SK, Chandrashekhar AP, Anjali S, Dhar M. Manual Liquid Based Cytology in Primary Screening for Cervical Cancer - a Cost Effective Preposition for Scarce Resource Settings. Asian Pacific Journal of Cancer Prevention, 2012; 3645-3651

[9] Hayama FH, Motta CF, Silva A, Migliari DA. Liquid-based preparations versus conventional cytology: specimen adequacy and diagnostic agreement in oral lesions. Med Oral Patol Oral Cir Buccal 2005;10:115-22.

[10] Sherwani RK, Khan T, Aktar K, et al (2007). Conventional pap smear and liquid based cytology for cervical cancer screening-A comparative study. J Cytology, 24, 167-72.

[11] Kavatkar AN, Nagwamshi CA, Dabaks M (2008). Study of a manual method of liquid based cervical cytology. Indian $\mathrm{J}$ Pathology and Microbiology, 59, 190-4.

[12] Delavarian Z, Mohtasham N, Mozaffari P, Pakfetrat A, Shakeri M, Maddah R. Evaluation of the diagnostic value of a Modified Liquid-Based Cytology using OralCDx ${ }^{\circledR}$ Brush in early detection of oral potentially malignant lesions and oral cancer. Med Oral Patol Oral Cir Buccal. 2010 Sep 1: 15 (5):e671-6.
[13] Maksem JA, Finnomore M, Belsheim BL, et al (2001). Manual method for liquid based cytology. A demonstration using 1,000 Gynaecological cytologies collected directly to vial and prepared by a smear - slide technique. Diagn Cytopathology, 25, 334-8.

[14] Rajendran R, Shivpathasundharam B. Shafer's Textbook of Oral Pathology. $6^{\text {th }}$ edition. New Delhi: Elsevier; 2009.

[15] Mehrotra R. Oral cytology. 12th ed. Springer; 2013. p.5.

[16] Sherwani RK, Khan T, Akhtar K, Zeba A, Siddiqui FA, Rahman K, Afsan N. Conventional Pap Smear and Liquid Based Cytology for Cervical Cancer Screening - A Comparative Study. Journal of Cytology 2007;24(4):167-172

[17] Deshou H, Changhua W, Quiyan L, et al (2009). Clinical utility of LiquiPrep cytology system for primary cervical cancer screening in a large urban hospital setting in China. $J$ Cytology, 26, 20-5.

[18] Johnson T, Maksem JA, Belshein BL, et al (2000). Liquid based cervical cell collection with brushes and wooden spatulas. Diagn Cytopathol, 22, 86-91.

[19] Vidal A K, Junior A, Mello R J, Brandão R A, Lima PA, Figueiroa J N. Conventional Cytology versus liquid-based cytology for prevention and early diagnosis of oral squamous cell carcioma (OSCC). Odontol. Clín.-Cient., Recife, 2011; 10 (1) $31-36$

[20] Navone R, Burlo P, Pich A, Pentenoro M, Broccoletti R, Marsico A et al. The impact of Liquid-based cytology on the diagnosis of oral squamous cell dysplasia and carcinoma. Cytopathology 2007;18:356-360. 